\title{
Simulation of nucleation and emission of dislocations by molecular- dynamics method
}

\author{
Y. W. Zhang, T. C. Wang, and Q. H. Tang \\ LNM. Institute of Mechanics, Chinese Academy of Sciences, Beijing 100080, People's Republic of China
}

(Received 12 September 1994; accepted for publication 22 November 1994)

\begin{abstract}
The nucleation and emission of dislocations from the crack tip under mode II loading are analyzed by the molecular-dynamics method in which the Finnis-Sinclair potential has been used. A suitable atom lattice configuration is employed to allow one to fully analyze the nucleation, emission, dissociation, and pileup of the dislocations. The calculated results show that although the pure mode II loading is applied, the crack tip generally exhibits a combined mode. The stress distributions before the dislocation emission are in agreement with the elasticity solution, but are not after the emission. The critical stress intensity factor corresponding to the dislocation nucleation $K_{\Pi l}$ is dependent on the loading rate $\dot{K}_{\Pi}$. The separations of a pair of partial dislocations and the full dislocations are also dependent on the loading rate. When the first partial dislocation is blocked, a pileup of dislocations can be set up. It is also found that the dislocation can move at subsonic wave speed (less than the shear wave speed) or at transonic speed (greater than the shear wave speed but less than the longitudinal wave speed) depending on the loading rate, but at the longitudinal wave speed which just corresponds to $\dot{K}_{\mathrm{II}}=1.15 \mathrm{MPa} \sqrt{\mathrm{m}} / \mathrm{ps}$ for copper, the atom lattice breaks down. (C) 1995 American Institute of Physics.
\end{abstract}

\section{INTRODUCTION}

The dislocation nucleations from the crack tip have been investigated by many researchers. ${ }^{1-4}$ Rice and Thomson ${ }^{2}$ used linear elasticity to establish the criterion of nucleation of a discrete dislocation and the transition of ductile and brittle fracture. Sinclair and Finnis ${ }^{5}$ extended Rice and Thomson analysis to address the problem of whether the presence of one or more emitted dislocation affects the competition between further emission and cleavage. Lin and Hirth $^{6}$ studied the changes of stress with dislocation emission from a crack. More recently, Rice $^{3}$ used the Peierls concept to reanalyze the dislocation nucleation from a crack tip and proposed a new solid-state parameter $\gamma_{u s}$, the unstable stacking energy, to evaluate the critical external loading which corresponds to dislocation nucleation. Wang ${ }^{4}$ extended Rice's analysis with a set of new governing equations which can be employed to analyze the dislocation emission.

The above analyses were unsatisfactory because of their use of the continuum elasticity for crack tip stress field. When a dislocation is very near the crack tip where the nonlinear and atomic lattice effects are great, the atomic force law needs to be considered. The experimental results of Choi et $a{ }^{7}{ }^{7}$ showed that the core size of dislocation is far beyond that predicted by the linear continuum elasticity theory. It is essential to analyze the dislocation nucleation and emission from the crack tip by atomistic simulation utilizing appropriate interatomic law and correct crystallographic geometry of the simulated specimen.

Bullough $^{8}$ and Cotterill and Doyama ${ }^{9}$ made what was probably the first such calculation. The potentials of BornMayer and Morse were used in their calculation. deDelis et al. ${ }^{10}$ used molecular dynamics with the Johnson and Morse potential to simulate stressed crack tip processes in $\alpha$-iron and copper.
Daw and Baskes ${ }^{11}$ developed the embedded-atom method to calculate the ground-state behaviors. Using the embedded-atom method, Baskes et $a{ }^{12}{ }^{12}$ investigated the dislocation mobility in nickel. Tan and Yang ${ }^{13}$ also used the embedded-atom method to calculate the nucleation and emission of dislocations at and near the crack tip. A new combined atomistic-continuum method was proposed. But a simplified atom lattice configuration was used in their calculation. Cheung et al. ${ }^{14}$ also used the embedded-atom method to analyze the dislocation nucleation from crack tips in $\alpha$-iron; their emphasis was put on the thermoactivity.

Here we use the " $N$-body" potential proposed by Finnis and Sinclair ${ }^{15}$ and constructed by Ackland et al. ${ }^{16}$ to simulate the crack tip processes in the ground state. The principal differences between the Finnis and Sinclair potential and the Daw and Baskes potential lie in the derivation and interpretation of electron density and the embedded function. Finnis and Sinclair used a square-root function for embedded function and identified electron density with the second moment of density of states.

Kitagawa and Nakatani ${ }^{17}$ used the Finnis and Sinclair potential to calculate the crack tip processes. Several atomistic lattice geometries and crack modes were employed to calculate the nucleation and emission of dislocations.

In order to investigate the dissociation, nucleation, emission, dislocation-free zone, and pileup of dislocations and compare them with the elasticity solution, a mode II crack under the prescribed $K$-displacement field is employed to analyze the crack tip processes in the present atomistic simulation. The calculated results obtained in the present paper can give us some comprehensive understanding about the nucleation, emission, dislocation-free zone, dissociation, and pileup of dislocations and some behaviors of moving dislocations. 
The plan of the paper is as follows. In the next section, we shall consider the atom model; these include (a) the interatomic potential, (b) the method of solution, (c) the atomistic geometry, and (d) the boundary conditions. In Sec. III, we shall give out our simulated results and compare them with the elasticity solutions. Finally, we shall give some conclusions in Sec. IV.

\section{ATOM MODEL}

\section{A. Interatomic potential}

The interatomic potential used here is the " $N$-body" potential proposed by Finnis and Sinclair. The ansatz they used is

$$
U_{\mathrm{tot}}=-\sum_{i} \rho_{i}^{1 / 2}+\frac{1}{2} \sum_{i} \sum_{i(i \neq j)} V_{i j}
$$

$\rho$ is the second moment of the density of states, and

$$
\rho_{i}=\sum_{j(i \neq j)} \Phi_{i j}
$$

$V_{i j}$ and $\phi_{i j}$ are functions only of the interatomic distance, and can be obtained by assuming some function forms and then fitting with the experimental data.

\section{B. Method of solution}

The mode II isotropic displacement field is prescribed to the border of discrete atoms for the initial positioning of atoms in an incremental fashion. The loading rate $\dot{K}_{\mathrm{IT}}$ is used as the loading control parameter. The inner atoms follow law of Newton:

$$
\mathbf{F}_{i}=-\frac{\partial U_{\text {tot }}}{\partial \mathbf{r}_{i}}=m_{i} \cdot \mathbf{a}_{i}
$$

In the present paper, the leapfrog algorithm is used:

$$
\begin{aligned}
& \mathbf{v}_{\mathbf{i}}\left(t+\frac{\Delta t}{2}\right)=\mathbf{v}_{\mathbf{i}}\left(t-\frac{\Delta t}{2}\right)+\frac{\mathbf{F}_{\mathbf{i}}}{m_{i}} \Delta t, \\
& \mathbf{r}_{\mathbf{i}}(t+\Delta t)=\mathbf{r}_{\mathbf{i}}(t)+\mathbf{v}_{\mathbf{i}}\left(t+\frac{\Delta t}{2}\right) \Delta t, \\
& \mathbf{v}_{\mathbf{i}}(t)=\frac{1}{2}\left[\mathbf{v}_{\mathbf{i}}\left(t+\frac{\Delta t}{2}\right)+\mathbf{v}_{\mathbf{i}}\left(t-\frac{\Delta t}{2}\right)\right],
\end{aligned}
$$

where $m_{i}$ is the mass of the $i$ th atom, and $\mathbf{v}_{\mathbf{i}}(t)$ and $\mathbf{r}_{\mathbf{i}}(t)$ the velocity and position of the $i$ th atom at time $t$, respectively. The above scheme provides an update formulation; the time step in the present calculation is taken to be $1.18 \times 10^{-14} \mathrm{~s}$.

The atomic level stress associated an atom is calculated by using the potential of Finnis and Sinclair:

$$
\sigma_{\alpha \beta}^{i}=\frac{1}{2 \Omega^{i}}\left[\sum_{j} V^{\prime}\left(r^{i j}\right)-\rho_{i}^{-1 / 2} \sum_{j} \Phi^{\prime}\left(r^{i j}\right)\right] \frac{r_{\alpha}^{i j} r_{\beta}^{i j}}{r^{i j}} \text {. }
$$

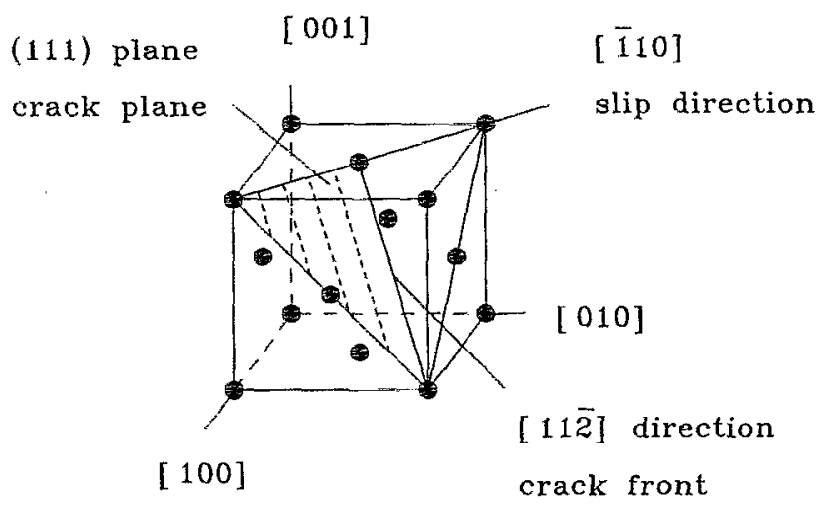

FIG. 1. Crack tip and crystallographic geometry in fcc crystal.

\section{The atom lattice geometry}

The $\{110\},\{111\}$, and $\{112\}$ crystallographic planes of the parallelepiped with a slit are used in present calculations. The coordinate system is selected to be $x, y$, and $z$ axes along the $\langle 110\rangle,\langle 112\rangle$, and $\langle 111\rangle$ directions, respectively. In fcc crystal, dislocation moves in the $\langle 110\rangle$ direction on the $\{111\}$ plane. So in the present model, the crack plane is taken to be $\{111\}$ plane and the crack front is along the $\langle 112\rangle$ direction. Under mode II loading, the dislocations shall move along the $\langle 110\rangle$ direction (see Fig. 1). The periodicity along $\langle 111\rangle$ is three layers, along $\langle 110\rangle$ is two layers, and along $\langle 112\rangle$ is six layers. Figure 2 shows the lattice atoms in our model on the $\{111\}$ plane. Since a long extension in slip direction is particularly important, here the $\langle 110\rangle$ slip direction is made as extensive as is computationably feasible.

A full dislocation in copper will be dissociated into two partial dislocations, i.e.,

$$
\frac{1}{2}[\overline{1} 10] \rightarrow \frac{1}{6}[\overline{2} 11]+\frac{1}{6}[\overline{1} 2 \overline{1}] .
$$

Between the two partial dislocations, there is a faulted plane. As for the periodicity condition is used along the $\langle 112\rangle \mathrm{di}-$ rection, the nucleation and motion of partial dislocations can be described with the present lattice configuration.

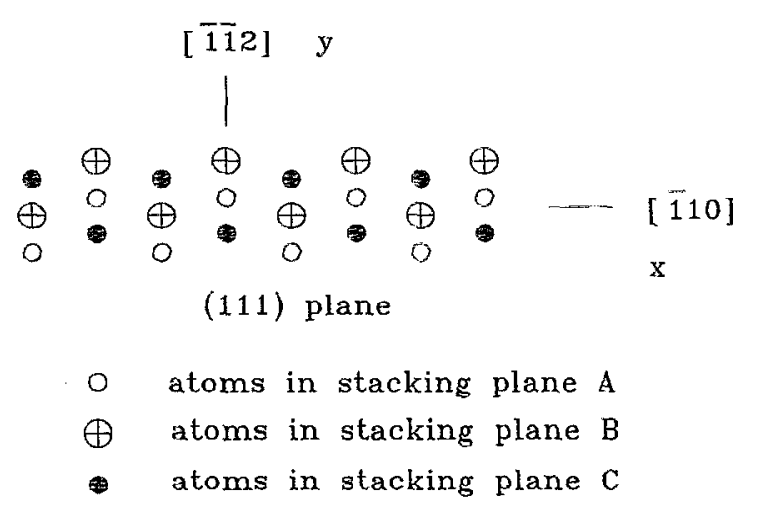

FIG. 2. The lattice atoms on the $\{111\}$ stacking plane. 
along x: 90 periods, 180 layers

along $y: 1$ periods 6 layers

along $z$ : 20 periods 60 layers

displacement boundaries

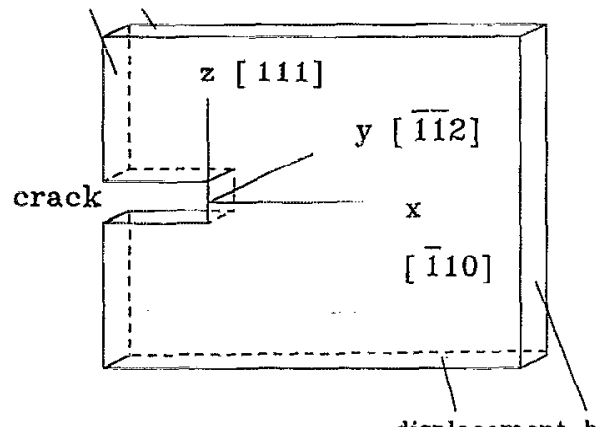

(a)

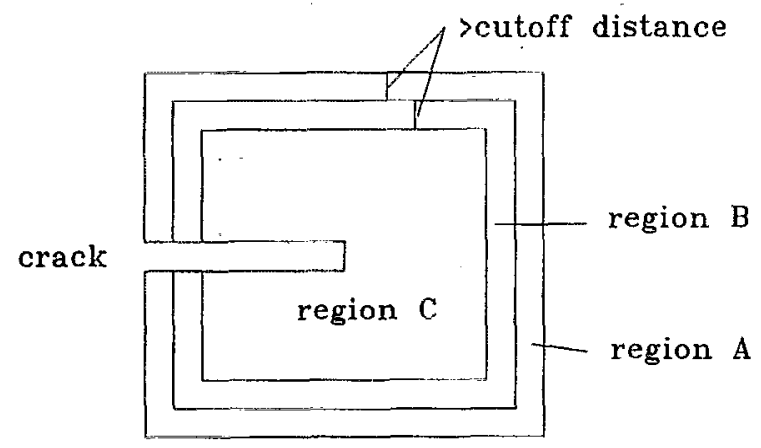

(b)

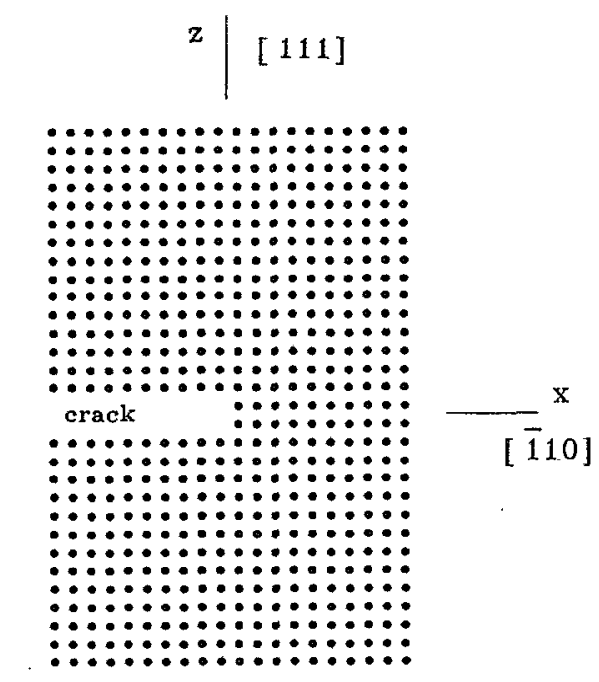

(c)

FIG. 3. (a) The schematic diagram of present calculated model, (b) the schematic diagram for the border treatment, and (c) the initial positioning of atoms near the crack tip, six layers projected at the $\{112\}$ plane.

\section{The boundary conditions}

The calculated model is schematically shown in Fig. 3(a). The boundary conditions applied to the boundaries of the discrete atom region A and B as shown in Fig. 3(b) have been that of a prescribed displacement distribution dictated by a model II isotropic $K$ field in the $x-z$ plane. The atoms in region $A$ are used to calculate the electron density of the atoms in region $\mathrm{B}$. The atoms in region $\mathrm{B}$ interact directly with the atoms in region $C$ governed by Newton's law. The width of regions A and B should be greater than the cutoff distance of the potential. Along the $y$ direction, a six layer periodic representation is applied. So the present atom lattice is actually three dimensional. Figure 3(c) shows the atom arrangement for copper of six adjacent $\{211\}$ planes projected on the same layer near the crack tip. The total number of atoms for the simulation is $N=10760$. The length along the $x$ direction is $(\sqrt{2} / 4) \times 180 a_{0}\left(a_{0}\right.$ is the lattice constant) and the width along $z$ direction is $(\sqrt{3} / 3) \times 60 a_{0}$. The distance between the crack tip and the left boundary is $(\sqrt{2} / 4) \times 20 a_{0}$. As the length from the crack tip to the right boundary is large enough, the effect of the boundary constraints on the nucleation and emission of dislocations can be neglected if the dislocations are not too close to the boundary. On the other hand, if we take the boundary as an obstacle to block the moving of dislocations, then the pileup of dislocations can then be set up.

\section{RESULTS AND DISCUSSIONS}

In the present paper, several cases under different loading rates have been calculated, but here mainly the case a loading rate, $\dot{K}_{\Pi}=0.02034 \mathrm{MPa} \sqrt{\mathrm{m}} / \mathrm{ps}\left(1 \mathrm{ps}=10^{-12} \mathrm{~s}\right)$, and case b, $\dot{K}_{\mathrm{II}}=0.05102 \mathrm{MPa} \sqrt{\mathrm{m}} / \mathrm{ps}$, are reported in detail. Then some other results obtained from other cases are also reported.

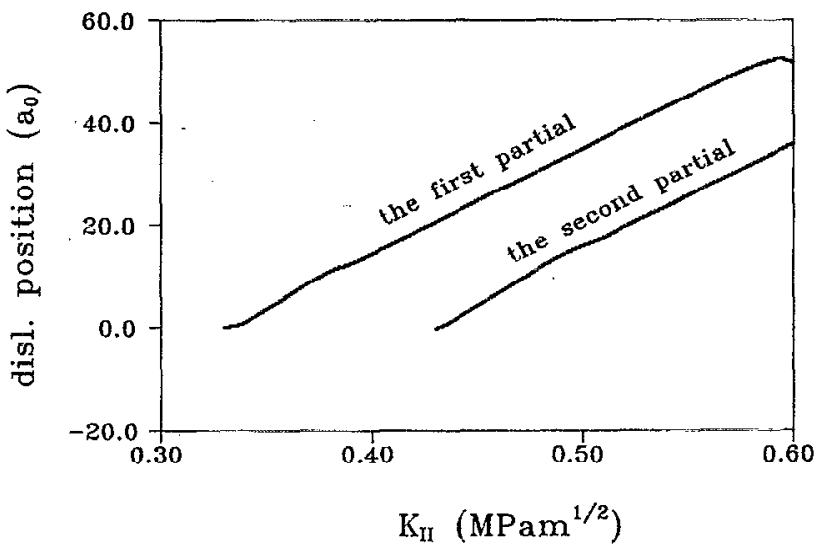

FIG. 4. The variations of positions of the partial dislocations with the isotropic loading level $K_{\mathrm{n}}$. Two partial dislocations have been emitted $\left(\dot{K}_{\mathrm{II}}=0.02034 \mathrm{MPa} \mathrm{m} / \mathrm{ps}\right)$. 


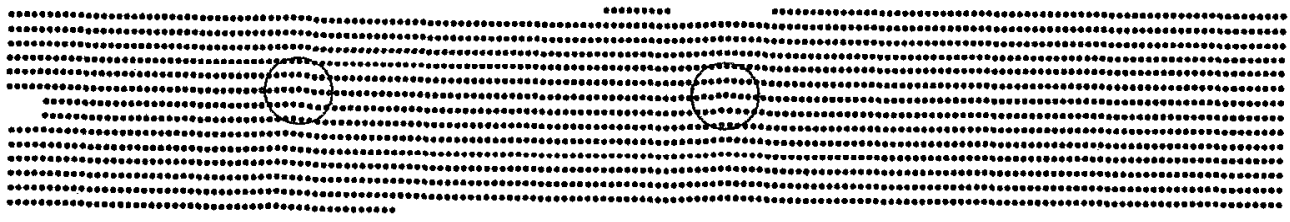

FIG. 5. The lattice atoms near the crack tip at $K_{\mathrm{I}}=0.48 \mathrm{MPa} \sqrt{\mathrm{m}}$. The two partial dislocations can be clearly observed.

\section{A. The calculated results for case a}

Although the pure mode II loading is applied, the moving-away direction is not along the $x$ direction, i.e., $\langle 110\rangle$, but along $30^{\circ}$ from the $x$ direction on the $x-y$ plane, i.e., $\langle 112\rangle$. The moving-away direction is just one of the partial dislocations. So the loading mode of the crack tip is a mixed one due to the dissociation of a full dislocation.

The first partial dislocation is nucleated at $K_{\mathrm{II}}=0.33$ $\mathrm{MPa} \sqrt{\mathrm{m}}$; it only takes $0.553 \mathrm{ps}$ for this partial dislocation to be accelerated to an approximate constant speed at $v=1812$ $\mathrm{m} / \mathrm{s}$. But at the distance $11.55 a_{0}$ from the crack tip, the dislocation changes its speed to about $1549 \mathrm{~m} / \mathrm{s}$. At $K_{\text {II }}$ $=0.43 \mathrm{MPa} \sqrt{\mathrm{m}}$, the second partial dislocation is nucleated; it accelerates very quickly to the speed of about $1816 \mathrm{~m} / \mathrm{s}$. At a distance $14.68 a_{0}$, its speed becomes approximately 1460 $\mathrm{m} / \mathrm{s}$. All these speeds are below the shear wave speed 2215 $\mathrm{m} / \mathrm{s}$ and longitudinal wave speed $4560 \mathrm{~m} / \mathrm{s}$ for copper. The separation between two partial dislocations is about $60 \AA$; it is well in the range predicted by Rice. ${ }^{3}$ Figure 4 shows curves of the dislocation positions versus loading level $K_{\text {II }}$. It can be seen that the separation of the two partial dislocations remains approximately the same except when the first dislocation is blocked by the border. When the first full dislocation is emitted, there exists a dislocation-free zone between the crack tip and the full dislocation. Figure 5 shows the atom configuration at $K_{\mathrm{II}}=0.48 \mathrm{MPa} \sqrt{\mathrm{m}}$. The two partial dislocations can be clearly observed. The shear stress along the prolongation of the crack plane is shown in Fig. 6(a) and 6(b) at loading level $K_{\mathrm{II}}=0.24$ and $0.48 \mathrm{MPa} \sqrt{\mathrm{m}}$. The shear stress of the elastic crack tip field and discrete dislocation (the shear stress at the dislocation core is corrected by Peierls dislocation) is also plotted on the same figure. The linear elastic solution of the interaction field produced by singularity of the crack tip and the edge dislocation with Burgers' vector $b$ is ${ }^{18}$

$$
\sigma_{x z}=\frac{K_{\mathrm{II}}}{\sqrt{2 \pi x}}+\sum_{i} \frac{\mu}{2 \pi(1-\nu)} \sqrt{\frac{x_{i}}{x}} \frac{b}{x-x_{i}} .
$$

The stress produced by Eq. (6) ensures no traction at the crack planes. The shear stress very near the dislocation core corrected by Peierls dislocation is ${ }^{19}$

$$
\sigma_{x z}=\frac{\mu b}{2 \pi(1-\nu)} \frac{x}{x^{2}+\zeta^{2}} .
$$

It is shown that before the dislocation nucleation, our results are in good agreement with the elasticity solution [see
Fig. 6(a)]. After the dislocation emission, our results are in disagreement with the elasticity solution [see Fig. 6(b)]. The stress level of our results is lower than the elasticity solution. This is because the stress of our results does not have singularity at the crack tip and the stress level at the dislocation core is lower than that of the Peierls dislocation due to the relaxation of the atoms at dislocation core.

\section{B. The calculated results for case $b$}

The curves of the dislocation positions versus loading level are shown in Fig. 7. As for the loading rate, case $b$ is larger than that of case a, the moving speed of dislocation is faster, and the separation of the two partial dislocations and the separation of the full dislocation are less than that of case
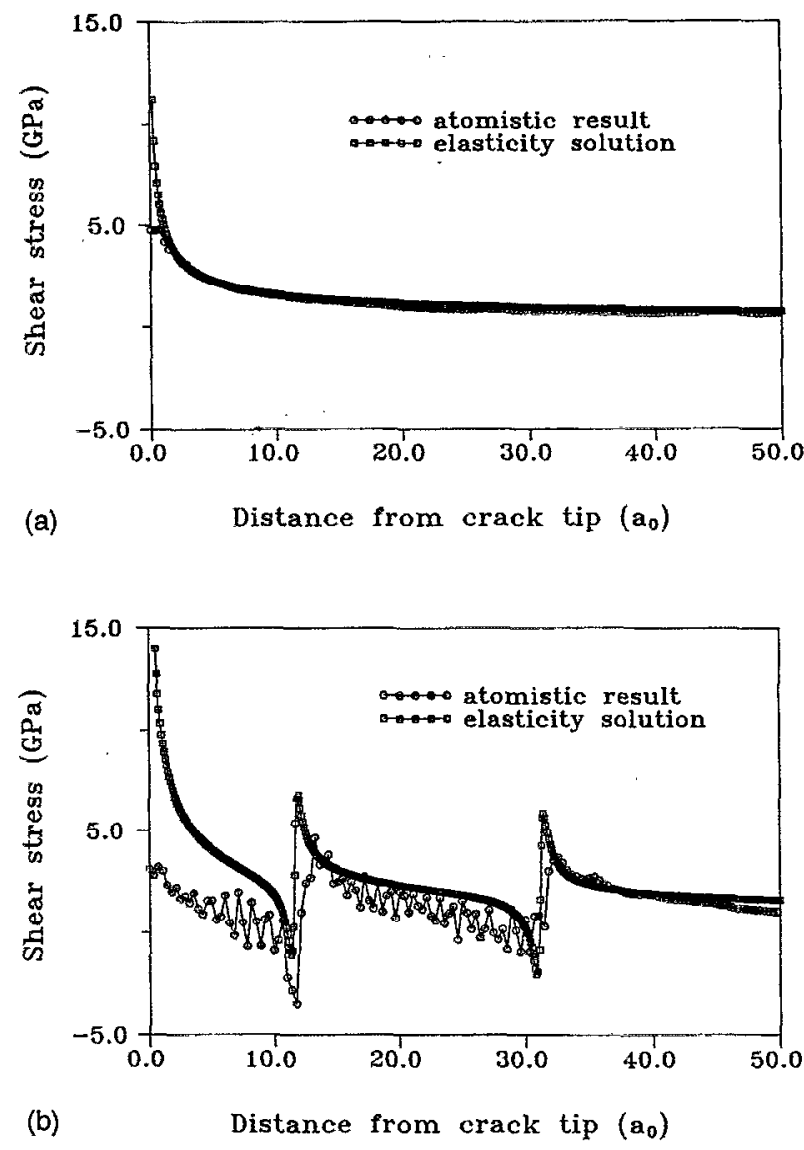

FIG. 6. The comparison of atomistic results and elasticity solutions, (a) before dislocation nucleation at $K_{\mathrm{II}}=0.24 \mathrm{MPa} \sqrt{\mathrm{m}}$ and (b) after dislocation emission at $K_{\mathrm{II}}=0.48 \mathrm{MPa} \sqrt{\mathrm{m}}\left(\dot{K}_{\mathrm{II}}=0.02034 \mathrm{MPa} \sqrt{\mathrm{m}} / \mathrm{ps}\right)$. 


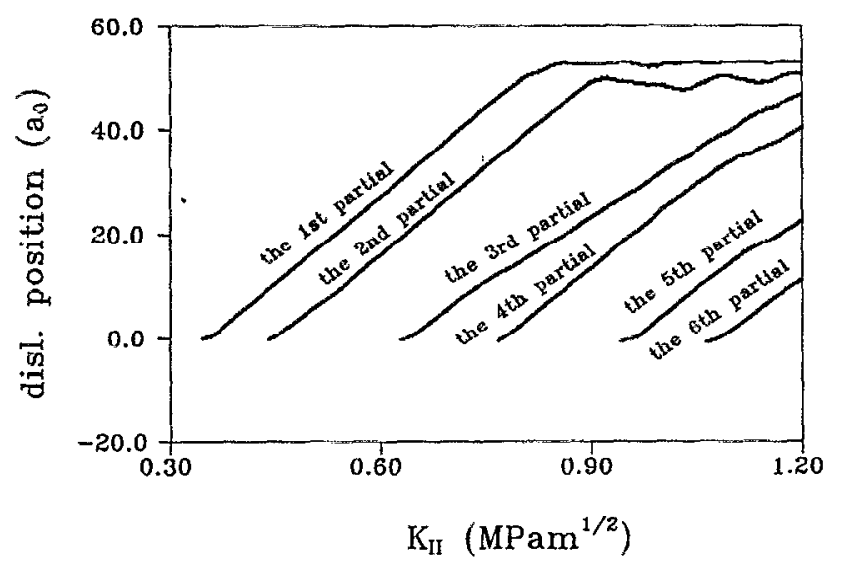

FIG. 7. The variations of the positions of the partial dislocations with the isotropic loading level $K_{\text {II. }}$. Six partial dislocations have been emitted $\left(\dot{K}_{\mathrm{U}}=0.05102 \mathrm{MPa} \sqrt{\mathrm{m}} / \mathrm{ps}\right)$.

a. The speed of the first partial dislocation remains approximately constant at $2123 \mathrm{~m} / \mathrm{s}$. When the first partial dislocation is blocked, it wanders near the border. When the following partial dislocation approaches the first blocked dislocation, it slows down and also wanders near the first partial dislocation. At this time the separation of these two partials is very close. It can be also noted that the dislocation-free zone at this case becomes smaller because of the increasing loading rate. The atom lattice configuration at $K_{\mathrm{II}}=1.2 \mathrm{MPa} \sqrt{\mathrm{m}}$ is shown in Fig. 8 . There are six partial dislocations between the crack tip and the border. The pileup of the dislocations can be clearly observed. The shear stress along the prolongation of the crack plane is shown in Figs. 9(a) and 9(b) at loading level $K_{\mathrm{II}}=0.24$ and $0.72 \mathrm{MPa} \sqrt{\mathrm{m}}$. $\mathrm{m}$. Comparison of our calculated results with the elasticity solution shows the same as that of case a.

\section{Some other results}

Our calculations also show that the edge dislocation can not only move at subsonic velocity, but also move at transonic velocity. At either the Rayleigh wave speed or shear wave speed, no special effects occur. But at the longitudinal wave speed, the atom lattice breaks down. The results are consistent with that of Weiner and Pear. ${ }^{20}$
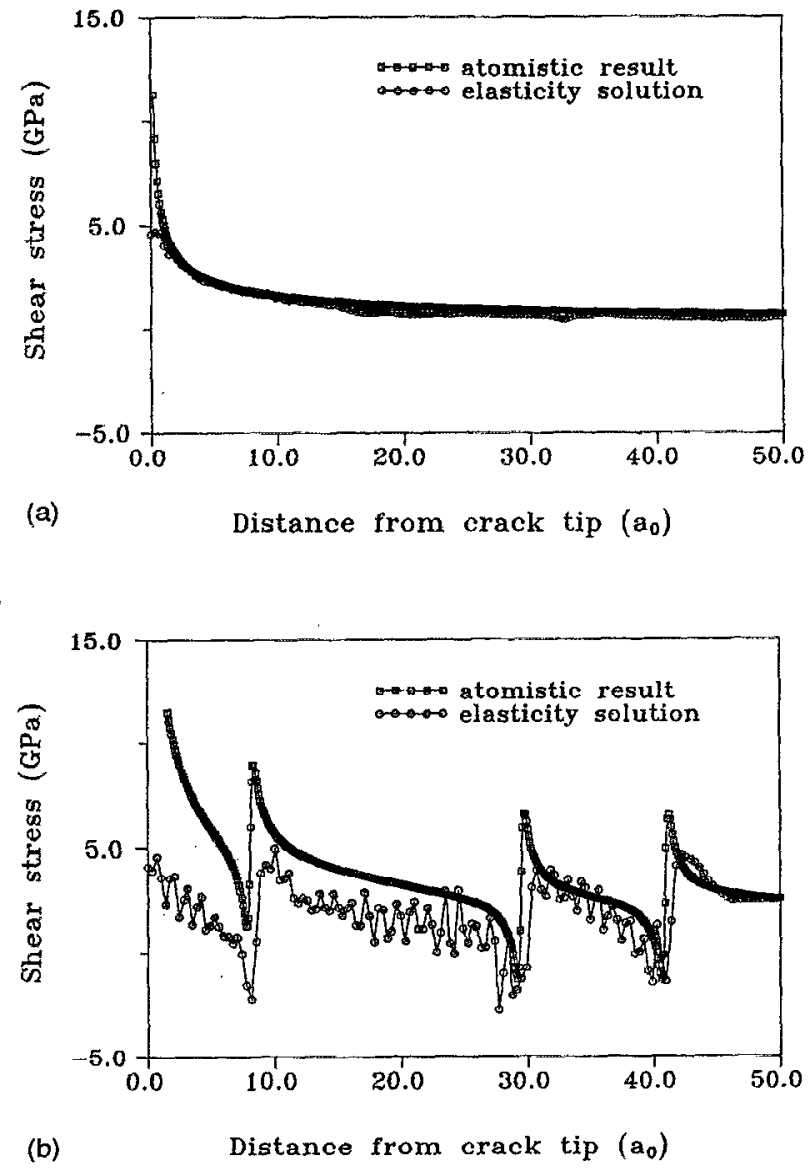

FIG. 9. The comparison of the atomistic results and clasticity solutions, (a) before dislocation nucleation at $K_{\mathrm{II}}=0.24 \mathrm{MPa} \sqrt{\mathrm{m}}$ and (b) after dislocation emission at $K_{\mathrm{I}}=0.72 \mathrm{MPa} \sqrt{\mathrm{m}}\left(\dot{K}_{\mathrm{II}}=0.05102 \mathrm{MPa} \sqrt{\mathrm{m}} / \mathrm{ps}\right)$.

The relation between the critical stress intensity factor of the first partial dislocation $K_{\text {II }}$ and loading rate $\dot{K}_{\text {II }}$ is shown in Fig. 10. We find that $K_{\text {IIe }}$ increases with the increase of $\dot{K}_{\text {II }}$. We also find that when $\dot{K}_{\text {II }}=1.15 \mathrm{MPa} \sqrt{\mathrm{m}} / \mathrm{ps}$, a partial dislocation is nucleated, but at same time, the atom lattice breaks down. The speed of the partial dislocation under the loading rate just corresponds to the longitudinal wave speed. The loading rate is the critical loading rate for transition from the ductile to brittle.

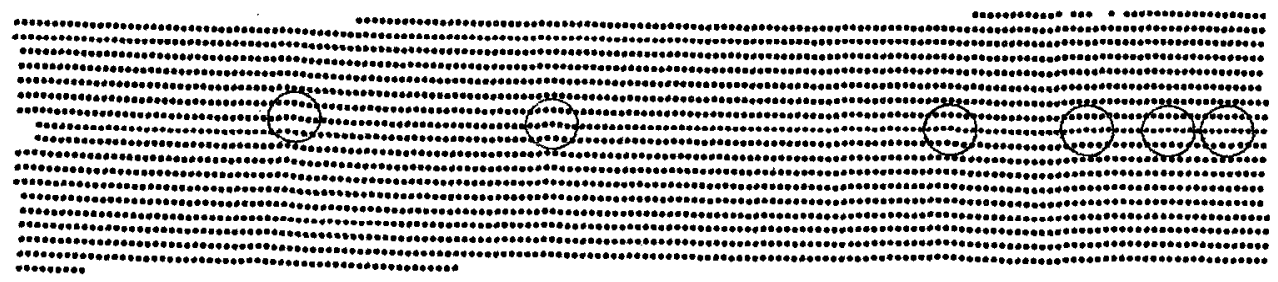

FIG. 8. The lattice atoms near the crack tip at $K_{\mathrm{n}}=1.2 \mathrm{MPa} \sqrt{\mathrm{m}}$. The six partial dislocations can be clearly observed. 


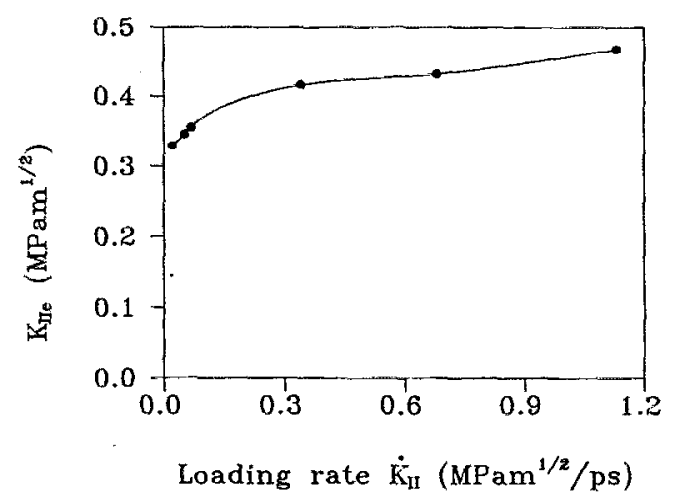

FIG. 10. The critical stress intensity factor of the first partial dislocation nucleation $K_{\mathrm{II} e}$ versus loading rate $\dot{K}_{\mathrm{II}}$.

At a higher loading rate, the separations of the partial dislocations and of full dislocations become very close. We also find that even at a lower loading rate, when the pileup of the dislocations develops at some $K_{\mathrm{II}}$, the atom lattice also breaks down. For example, when loading rate $\dot{K}_{\mathrm{II}}=0.0638$ $\mathrm{MPa} \sqrt{\mathrm{m}} / \mathrm{ps}$, is applied, atom lattice breaks down at $K_{\mathrm{II}}=1.36 \mathrm{MPa} \sqrt{\mathrm{m}}$. But at same loading rate $\dot{K}_{\mathrm{II}}$ and loading level $K_{\mathrm{II}}$ the failure does not take place when we enlarge the length along the $x$ direction.

Figure 11 shows the partial dislocation core structure. The core structure is different from that of the discrete dislocation and the Peierls dislocation. It can be seen that the relaxation of core atoms takes place due to the high stress level at the dislocation core.

It should be noted that there are several aspects which may influence the present calculated results. The first one is the inappropriate boundary condition of using the isotropic displacement field. The work of Cheung et al. ${ }^{14}$ shows that the effect of the anisotropic $K$-displacement field may reduce

six layers projected on (112) plane

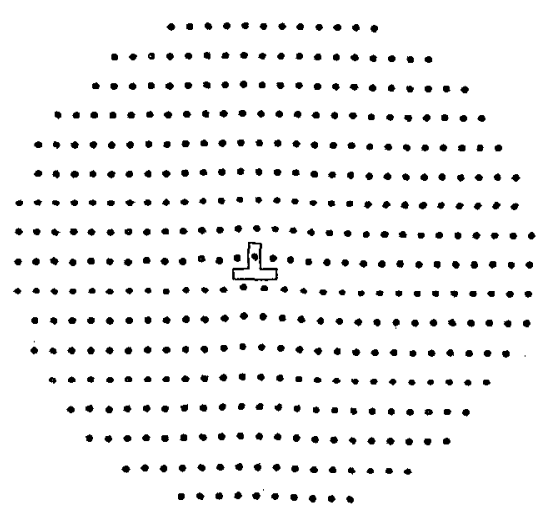

FIG. 11. The atom configuration at the dislocation core. The relaxation of atoms can be observed.

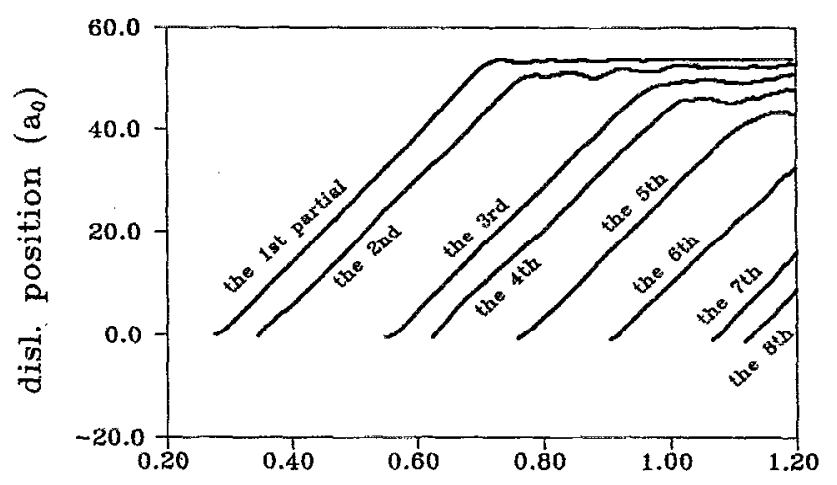

$\mathrm{K}_{\text {II }}\left(\mathrm{MPam}^{1 / 2}\right)$

FIG. 12. The variations of the positions of the partial dislocations with the anisotropic loading level $K_{\mathrm{II}}$. Eight partial dislocations have been emitted $\left(\dot{K}_{\mathrm{II}}=0.05102 \mathrm{MPa} \sqrt{\mathrm{m} / \mathrm{ps}}\right)$.

the critical stress intensity factor for the crack propagation. Our preliminary work of using the anisotropic field does show that the critical value for the dislocation emission is reduced by $24 \%$ and the more dislocations are emitted at the same loading level as shown in Fig. 12. But our work also shows that the use of the anisotropic displacement boundary condition does not change the overall qualitative features of the crack tip processes of using the isotropic displacement boundary condition. The second aspect is the size effect. The narrowest distance from the crack tip to the boundary along the $z$ direction in the present calculation is $(\sqrt{2} / 4) \times 30 a_{0}$ $\left(a_{0}=3.615 \AA\right)$. We enlarge the distance along the $z$ direction; the calculated results show no apparent difference. So the size effect along the $z$ direction in the present simulation is comparatively small. This is in agreement with the discussions made by McCoy and Markworth ${ }^{21}$ and Hoagland et al., ${ }^{22}$ who show that diameters greater than $4 \mathrm{~nm}$, of the atom region around the crack tip, appear adequate.

\section{CONCLUSIONS}

The present molecular-dynamics simulation of crack tip processes can give us several insights of nucleation, emission, dissociation, and pileup of dislocations and the behaviors of moving dislocations.

(1) The critical stress intensity factor for the first partial dislocation $K_{\Pi l}$ is associated with the loading rate; the higher the loading rate, the higher the $K_{\text {IIe }}$. For copper, the critical loading rate for transition from ductile to brittle is $\dot{K}_{\Pi}=1.15 \mathrm{MPa} \sqrt{\mathrm{m}} / \mathrm{ps}$.

(2) The stress fields are consistent with the elastic stress field before the dislocation nucleation, but inconsistent with the elastic stress field plus discrete dislocations (stress at the dislocation core is corrected by the Peierls dislocation field stress) after the dislocation emission, and the dislocation core structure is not the same as the discrete dislocation and the Peierls dislocation because of the relaxation of atoms at the dislocation core. 
(3) For copper, a full dislocation always dissociates into two partial dislocations. The separation of the two partial dislocations is smaller than that of the full dislocations, and both depend on the loading rate.

(4) The dislocation can move at subsonic speed and transonic speed depending on the loading rate. When dislocation moves at longitudinal wave speed, the atom lattice breaks down; this means that the brittle failure occurs.

(5) The present boundary condition can be used to simulate the pileup of the dislocations. The pileup of dislocations increases the $K_{\mathrm{II} e}$ because of the back stress of the pileup of dislocations. The brittle failures may occur due to the high $K_{\text {Ie }}$. Here the failure exhibits the breakdown of the atom lattice.

(6) The size of the dislocation-free zone depends on the loading rate. The higher the loading rate, the smaller the size of the dislocation-free zone.

\section{ACKNOWLEDGMENTS}

The project is supported by the National Natural Science Foundation of China. We are grateful to Professor Zhu Fuxin and $M$. Sc. Zhong Jun for stimulating discussions.
${ }^{1}$ R. Thomson, Solid State Phys. 39, 1 (1986).

${ }^{2}$ J. R. Rice and R. Thomson, Philos. Mag. 29, 73 (1974).

${ }^{3}$ J. R. Rice, J. Mech. Phys. Solid 40, 239 (1992).

${ }^{4}$ T. C. Wang, Int. J. Fract. (in press).

${ }^{5}$ J. E. Sinclair and M. W. Finnis, in Atomics of Fracture, edited by R. M. Latanision and J. R. Pickens (Plenum, New York, 1983).

${ }^{6}$ I. H. Lin and J. P. Hirth, Philos. Mag. A 50, L43 (1984).

${ }^{7}$ H. C. Choi, A. F. Schwartzman, and K. S. Kim, Mater. Res. Soc. Symp. Proc. 239, 419 (1992).

${ }^{8}$ R. Bullough, Bull. Am. Phys. Soc. 10, 323 (1965).

${ }_{9}^{9}$ R. M. J. Cotterill and M. Doyama, Phys. Rev. 145, 465 (1966).

${ }^{10}$ B. deCelis, A. S. Argon, and S. Yip, J. Appl. Phys. 54, 4864 (1983).

${ }^{11}$ M. S. Daw and M. I. Baskes, Phys. Rev. B 29, 6443 (1984).

${ }^{12}$ M. I. Baskes, M. S. Daw, and S. M. Foiles, Mater. Res. Soc. Symp. Proc. 141, 31 (1989).

${ }^{13}$ H. L. Tan and W. Yang, Acta Mech. Sinica 10, 150 (1994).

${ }^{14}$ K. S. Cheung, A. S. Argon, and S. Yip, J. Appl. Phys. 69, 2088 (1991).

${ }_{15}^{15}$ M. W. Finnis and J. E. Sinclair, Philos. Mag. 50, 45 (1984).

${ }^{16}$ G. J. Ackland, G. Tichy, V. Vitek, and M. W. Finnis, Philos. Mag. A 56, 735 (1987).

${ }^{17}$ H. Kitagawa and A. Nakataní, Proc. JSME Ser. A 59-557, 256 (1993).

${ }^{18}$ S. M. Ohr, J. Phys. Chem. Solids 48-11, 1007 (1987).

${ }^{19}$ R. E. Peierls, Proc. Phys. Soc. London 52, 34 (1940).

${ }^{20}$ See J. Weertman and J. R. Weertman, in Dislocations in Solids, edited by F. R. N. Nabarro (North-Holland, Amsterdam, 1980), Vol. 3.

${ }^{21}$ J. K. McMoy and A. J. Markworth, Scr. Metall. 20, 905 (1986).

${ }^{22}$ R. G. Hoagland, M. S. Daw, S. M. Foiles, and M. I. Baskes, J. Mater. Res. 5, 313 (1990). 